\title{
Perception of heading without retinal optic flow
}

\author{
KRISTEN L. MACUGA, JACK M. LOOMIS, ANDREW C. BEALL, and JONATHAN W. KELLY \\ University of California, Santa Barbara, California
}

\begin{abstract}
How do we determine where we are heading during visually controlled locomotion? Psychophysical research has shown that humans are quite good at judging their travel direction, or heading, from retinal optic flow. Here we show that retinal optic flow is sufficient, but not necessary, for determining heading. By using a purely cyclopean stimulus (random dot cinematogram), we demonstrate heading perception without retinal optic flow. We also show that heading judgments are equally accurate for the cyclopean stimulus and a conventional optic flow stimulus, when the two are matched for motion visibility. The human visual system thus demonstrates flexible, robust use of available visual cues for perceiving heading direction.
\end{abstract}

The ability to control our direction of motion (heading) is considered important for guiding locomotion. Gibson $(1950,1958)$ first proposed that locomotor behaviors could be carried out on the basis of the changing angular positions of visible environmental points produced by relative motion between the observer and the environment (optic flow). Psychophysical experiments have demonstrated that we are quite adept at judging heading with simulated optic flow. Threshold accuracies of $1^{\circ}-2^{\circ}$ have been reported (Cutting, Springer, Braren, \& Johnson, 1992; Royden, Banks, \& Crowell, 1992; van den Berg, 1993; Warren \& Hannon, 1988; Warren, Morris, \& Kalish, 1988). From physiological studies, we know that in the medial superior temporal (MST) area, many neurons are selective for optic flow (Saito et al., 1986; Tanaka et al., 1986) and that microstimulation of the MST area biases heading perception (Britten \& van Wezel, 1998). Other distinct areas are thought to be involved (for reviews, see Bremmer, Duhamel, Hamed, \& Graf, 2000, as well as Raffi \& Siegel, 2004). The majority of this physiological research has used simulated optic flow presented as random dot stimuli devoid of any depth information from disparity.

However, recent evidence that heading judgments of noisy monocular flow are more robust when static stereoscopic cues are included (van den Berg \& Brenner, 1994) indicates that depth information from disparity may be important, though only when there is ambiguity in the optic flow. Another intriguing finding is the enhancement of selfmotion perception with the addition of stereoscopic motion cues (Palmisano, 1996). The finding that perceived 3-D shape influences perceived heading (Beusmans, 1998) also suggests that depth information may contribute to head-

This work was supported by Air Force Office of Scientific Research Grant F49620-02-1-0145. A preliminary version of this research was presented at the Vision Sciences Society meeting, May 2003, in Sarasota, Florida. Correspondence should be addressed to K. L. Macuga, Department of Psychology, University of California, Santa Barbara, CA 93106-9660 (e-mail: macuga@psych.ucsb.edu). ing perception. Disparity-dependent direction-selective cortical neurons are capable of signaling the direction of self-motion (Roy, Komatsu, \& Wurtz, 1992; Roy \& Wurtz, 1990). Furthermore, perception of visual expansion does not require retinal optic flow (Schrater, Knill, \& Simoncelli, 2001), and coarse directional judgments are possible using sequentially presented, static scenes photographed from two different viewing positions (Hahn, Andersen, \& Saidpour, 2003). Here, by using a cyclopean stimulus (a random dot cinematogram), we demonstrate heading perception without retinal optic flow. We also show that heading perception is equally accurate for the cyclopean stimulus and a conventional optic flow stimulus when the two are matched for motion visibility.

To test whether heading can be perceived even when retinal optic flow is absent, we used a random dot cinematogram technique (Julesz, 1971) to generate a purely cyclopean stimulus to simulate observer motion through a 3-D environment. Binocular disparity information is produced by laterally displacing points displayed to one eye relative to those displayed to the other eye without changing the random structure of each monocular image. Changes in binocular disparities due to depth variations in a simulated environment can thus indicate observer motion. On every graphics frame, a stereogram is dynamically generated from a new sample of random dots. Accordingly, each eye individually sees only a scintillating array of random dots, since individual dots maintain no coherent motion. Observer motion is therefore camouflaged to monocular viewing, though motion through a depth-defined environment is quite apparent when viewed with both eyes. Others (Donnelly, Bowd, \& Patterson, 1997; Gray \& Regan, 1996; Portfors-Yeomans \& Regan, 1996) have used similar stimuli to investigate the binocular perception of object motion (see Patterson, 1999, for a review). Discrimination thresholds for direction of motion within the frontoparallel plane are the same for cyclopean and monocular targets when their visibilities are equated by presenting the motion displays at equal multiples of detection threshold (Donnelly et al., 1997). 
We investigated the degree to which heading judgments performed with the cyclopean stimulus would match the same judgments performed with a traditional stimulus that produces retinal optic flow. It is conceivable that if the visibilities for these two stimuli were equated, heading performance would be equated. Such a functional equivalence would indicate that a more abstract form of flow, not retinal optic flow, is being used to determine heading. Instead of being the primary determinant of perceived heading, retinal optic flow information may be one source of information that combines with other sources (such as binocular disparity information), to enable accurate heading judgments and ultimately locomotor behavior.

\section{EXPERIMENT 1 Heading}

The purpose of this experiment was to obtain heading thresholds using the cyclopean stimulus, and to compare these thresholds with a traditional optic flow stimulus. We presented computer displays of observer translation parallel to a ground plane covered with bumps (Figure 1), and had each observer make psychophysical judgments as to whether he or she was heading to the left or to the right of a probe that appeared on the final frame of each trial. Heading angle is defined as the angle between the heading direction and the direction of the probe.

\section{Method}

Stimuli. Displays simulated translational self-motion parallel to a horizontal ground plane. Bumps, approximately $1 \mathrm{~m}$ in diameter, were randomly placed along the ground plane and translated in depth toward the observer at a simulated speed of $6 \mathrm{~m} / \mathrm{sec}$. At any one time, 16-20 bumps were visible on a ground plane that extended out to a simulated distance of $20 \mathrm{~m}$ from the observer. Computer-generated images were presented in real time on a Virtual Research V8 LCDbased head-mounted display, driven by a $3.0 \mathrm{GHz} \mathrm{PC}$ and an nVidia Geforce 6800 graphics card. Each LCD panel (one for each eye) and associated condensing lens provided $640 \times 480$ pixels, covering a $50^{\circ}$ horizontal $\times 38^{\circ}$ vertical field of view with $100 \%$ binocular overlap, and were refreshed at $60 \mathrm{~Hz}$. Head position was fixed, but eye movements were allowed.

To specify the direction of translational self-motion with an optic flow stimulus, an optic flow pattern, consisting of small, randomly placed bumps on a ground plane, moved radially outward from the focus of expansion. Thus, this stimulus contained smooth optic flow. Figure 1A illustrates a static frame of optic flow stimulus. The cyclopean stimulus consisted of a sequence of random dot stereograms with single-frame lifetimes and depicted the same randomly placed bumps as in the optic flow stimulus on each frame. Thus, the disparity was consistently increasing in magnitude as the bumps moved radially outward. Figure 1B shows a single frame of the cyclopean stimulus. Note that these bumps are visible only binocularly. The cyclopean stimulus contained perspective images of 1,000 random dots rendered separately for the two eyes and then sent to the corresponding visual displays. The dots were spread over the lower half of the field of view with constant angular density. When viewed with both eyes, the cyclopean display revealed a changing 3-D stimulus, but when viewed by one eye alone, it appeared only as a scintillat-

\section{A}

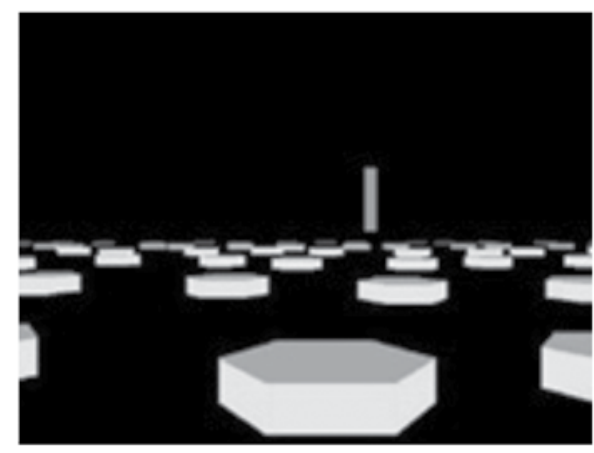

B

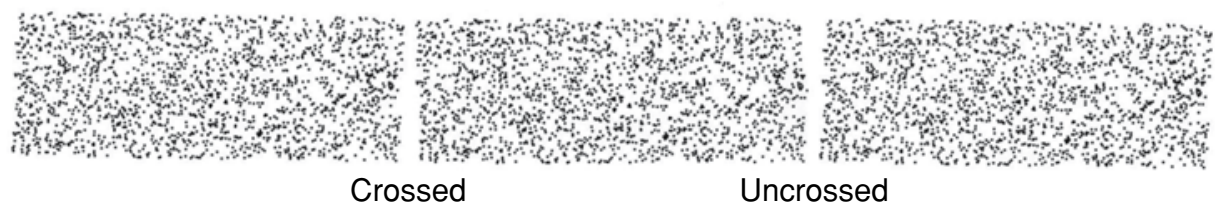

Figure 1. Static view of the ground plane used to simulate translational self-motion. (A) Optic flow stimulus. The final frame of a trial is depicted; note the target line probe. The target probe was not present during the simulated self-motion. (B) Random dot stereogram depicting the cyclopean stimulus (use left pair for crossed and right pair for uncrossed viewing). The actual cyclopean stimulus was made up of many frames like this one, presented dichoptically to the observer, simulating translational self-motion. The target line probe (not shown here) appeared in the last frame of the display, as it did in the optic flow stimulus. 
ing display of uniform dot density. Thus, there was no task-relevant optic flow signal in the cyclopean stimulus.

Design and Procedure. Four observers with $80 \%$ or better stereoacuity thresholds and 20/20 visual acuity, as measured on a Keystone orthoscope, participated in both the optic flow and scintillating random dot cinematogram conditions described above. To measure heading thresholds, we employed a discrimination task using the method of constant stimuli. Prior to the experiment, observers were given 10 practice trials with feedback to familiarize themselves with the task. Each experimental trial consisted of a $3.7-\mathrm{sec}$ presentation interval of a simulated heading direction. In the final frame of each trial, motion ceased, and a $0.25^{\circ}$ wide target line appeared on the horizon in one of four horizontal positions, $\pm 2^{\circ}$ and $\pm 6^{\circ}$ from the center of the display. The observers made a judgment as to whether they were heading to the right or to the left of the target line by pressing one of two response keys. The subjects were not given feedback about their responses. Heading angle between the heading direction and the target line was chosen randomly from one of 10 possible values $\left( \pm 0.5^{\circ}, \pm 1.0^{\circ}, \pm 2.0^{\circ}, \pm 4.0^{\circ}\right.$, or $\pm 6.0^{\circ}$ to the left or to the right of the target), with one trial for each combination of target position $\times$ heading angle. Therefore, the direction of heading could occur within a range of positions $\pm 12^{\circ}$ from the center of the display. Observer 1 had slightly different possible values $\left( \pm 0.2^{\circ}\right.$ included instead of $\pm 6^{\circ}$ initially). This observer's thresholds were quite low, but subsequent observers had some trouble on the practice trials, so we increased our range of possible values to those listed above. The observers alternated between the optic flow and cyclopean conditions on each trial set. For each display condition, the data were collapsed across target line positions and positive-negative heading angles. Psychometric functions were fit to the data, and mean thresholds of $75 \%$ correct were calculated.

\section{Results and Discussion}

Observers were quite accurate at judging their heading direction from both types of displays. Performance accuracy was measured using mean percent correct. In Figure 2, mean percent correct performance, averaged over 4 observers, is plotted for each absolute heading angle. Chance performance corresponds to $50 \%$ correct. The heading angle corresponding to $75 \%$ correct repre-

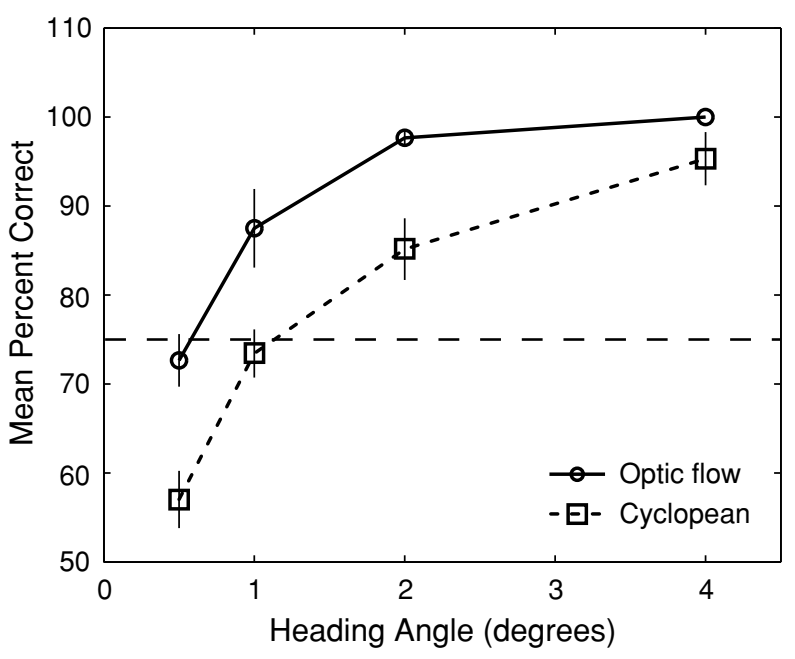

Figure 2. Mean percent correct for 4 observers in the optic flow and cyclopean conditions (collapsed across positive and negative heading angles). The dashed line represents $75 \%$ threshold. Vertical bars indicate standard errors. sents threshold performance. Error bars represent \pm 1 standard error of the mean. Heading angles of $\pm 6^{\circ}$ and $\pm 0.2^{\circ}$ are not plotted in this figure, since not all observers participated in these conditions. Observers performed the task with a slight difference in accuracy. Although the mean heading threshold was remarkably low $\left(1.35^{\circ}\right)$ for the cyclopean stimulus, it was reliably larger than the $0.68^{\circ}$ threshold for the optic flow stimulus [t(3) $=6.09, p<.01]$. Several observers reported that bump visibility was lower in the cyclopean condition. Thus, two caveats due to our display limitations deserve to be mentioned. The first is that our head-mounted display has a rather low display resolution for displaying cyclopean stimuli (horizontal pixel spacing of 4.5' of arc). The second is that the cyclopean display can currently be rendered with only 1,000 random dots in each graphics frame. As such, distant objects are defined by very few dots, causing them to be quite difficult to discern when depicted stereoscopically. Thus, visibility of small, distant objects is reduced for cyclopean viewing. Comparisons between psychophysical judgments of cyclopean and luminance-defined stimuli are compelling only when the different types of stimuli can be matched for visibility.

\section{Subsidiary Experiment for Matching Motion Visibility}

Therefore, we performed an experiment aimed at equating motion visibility for the optic flow and cyclopean stimuli. We fashioned this experiment after a control study done by Gray and Regan (1996) to equate cyclopean and monocularly visible targets for stimulus strength. Motion visibility for the optic flow stimulus was to be equated to motion visibility for the cyclopean stimulus by setting the target/background contrast to a value that effectively matched motion detection performance in both conditions. This contrast value was determined by the following subsidiary experiment. Observers 1-4 from the heading experiment, plus an additional observer, participated in this experiment.

The subsidiary experiment consisted of two parts. For both parts of the subsidiary experiment, a target disk in front of a background surface was sinusoidally oscillated in the frontoparallel plane. The first part measured the threshold amplitude required to detect movement of a sinusoidally oscillating cyclopean stimulus. The second part established the threshold contrast required to detect movement of an oscillating luminance-defined stimulus using the amplitude obtained from the first part.

In the first part, we established the threshold amplitude for discriminating cyclopean frontoparallel motion at two different frequencies. A disk subtending $11^{\circ}$ of visual angle in front of a background surface with a disparity of $0.1^{\circ}$ was sinusoidally oscillated in the frontoparallel plane at two frequencies: $0.5 \mathrm{~Hz}$ and $2.0 \mathrm{~Hz}$. A temporal twoalternative forced choice for amplitudes of $0.05^{\circ}-1.5^{\circ}$ of visual angle was used to get $75 \%$ correct motion detection thresholds. Each trial consisted of two randomly presented intervals. A stationary disk appeared in one interval, and a moving disk oscillating in the frontoparallel plane at 
one of six possible amplitudes appeared in the other interval. The duration of each interval was $2 \mathrm{sec}$, and the interstimulus interval was $0.5 \mathrm{sec}$. The observer indicated whether the moving disk was in the first or second interval. The mean percent correct responses were calculated and plotted against the test amplitudes. The discrimination threshold - the amplitude that was detectable $75 \%$ of the time - was estimated from the psychometric function by using probit analysis. Cyclopean frontoparallel oscillation thresholds were similar for both frequencies tested, with a mean threshold amplitude of $0.3^{\circ}$ and a standard deviation of $0.1^{\circ}$ for the $2-\mathrm{Hz}$ oscillation and a mean threshold amplitude of $0.5^{\circ}$ and a standard deviation of $0.1^{\circ}$ for the $0.5-\mathrm{Hz}$ oscillation frequency.

For the second part, we repeated the experiment with a luminance-defined disk, but this time we varied target/ background contrast while holding amplitude constant at the cyclopean frontoparallel motion threshold amplitude obtained in the first part. The disk once again subtended $11^{\circ}$ of visual angle and oscillated in the frontoparallel plane but was luminance defined rather than cyclopean. A spot meter was used to record values for both target and background luminance from the LCD panels of the head-mounted display. For a given stimulus, contrast was calculated as (maximum luminance - minimum luminance) / (maximum luminance + minimum luminance). The procedure and parameters were the same as for the previous portion of the experiment, except this time we varied target/background contrast between $1 \%$ and $7 \%$, while holding amplitude constant at the cyclopean threshold value for each frequency. The mean percent correct responses were calculated and plotted against target/background contrast.

We calculated the threshold contrast by fitting a probit function to the data. Contrast thresholds for frontoparallel oscillations were nearly identical for both oscillation frequencies tested. Threshold contrasts were also highly similar across observers. The threshold contrast for detecting target motion was approximately $4.4 \%$ for 2 observers, $4.9 \%$ for 2 observers, and $5.2 \%$ for 1 observer. If we set target/background contrasts to these values, the oscillatory motions of the contrast-defined and cyclopean disks moving with the same amplitude and frequency of motion were equally detectable. Thus, each observer's $75 \%$ threshold contrast was consequently used to match his or her optic flow condition to the cyclopean condition for motion visibility in the second heading experiment. Therefore, any differences between thresholds for optic flow and cyclopean stimuli should not be due to a mismatch between visibilities of the respective displays.

\section{EXPERIMENT 2 Heading With Equated Motion Visibility}

After matching the stimuli for motion visibility, as described above, we replicated the original heading experiment.

\section{Method \\ Stimuli. Except for the modified contrast in the optic flow con- dition, displays and task were otherwise identical to those in the}

original heading experiment. Figure 3 depicts the modified-contrast optic flow stimulus. The print version may differ slightly from the actual stimulus because of distortions in the reproduction process.

Design and Procedure. Observers 1-4 plus an additional observer participated in the equated motion visibility heading experiment. Heading angle between the heading direction and the target line was chosen randomly from one of 10 possible values $\left( \pm 0.5^{\circ}, \pm 1.0^{\circ}\right.$, $\pm 2.0^{\circ}, \pm 4.0^{\circ}, \pm 8.0^{\circ}$ to the left or to the right of the target). We also included a manipulation of the simulated translation speed of the observer $(2 \mathrm{~m} / \mathrm{sec}$ and $6 \mathrm{~m} / \mathrm{sec}$ ) in this experiment. Cutting et al. (1992) reported calculations of heading accuracy necessary for different speeds of locomotion (i.e., walking, jogging, sprinting, etc.). Based on some assumptions, required accuracies range from $3.7^{\circ}$ for $2 \mathrm{~m} / \mathrm{sec}$ to $0.7^{\circ}$ for $10 \mathrm{~m} / \mathrm{sec}$. The questions of interest were: Does the cyclopean condition allow for such accuracies across speed manipulations, and how does it compare to the optic flow condition? Displays were blocked by speed and presented in a counterbalanced order.

\section{Results and Discussion}

Mean percent correct performance is plotted in Figure $4 \mathrm{~A}$ as a function of stimulus type and simulated speed over the ground plane. Figure 4B shows threshold values for each subject. The results once again demonstrate that heading direction can be judged with remarkable accuracy with the cyclopean stimulus. Mean thresholds were $1.1^{\circ}$ for optic flow simulated at $6 \mathrm{~m} / \mathrm{sec}, 1.6^{\circ}$ for optic flow simulated at $2 \mathrm{~m} / \mathrm{sec}, 1.6^{\circ}$ for the cyclopean stimulus simulated at $6 \mathrm{~m} / \mathrm{sec}$, and $1.7^{\circ}$ for the cyclopean stimulus simulated at $2 \mathrm{~m} / \mathrm{sec}$. As evidenced by the data, with the reduced contrast, the performance for cyclopean stimuli was very similar to the performance for optic flow stimuli, especially in the slower speed conditions. Compared to performance in the prior experiment, reducing contrast raised overall thresholds for optic flow. Although it might appear that there is a speed effect occurring in the optic flow condition (see Figure 4C), a within-subjects analysis of variance on mean thresholds revealed no main effect of display condition $[F(1,3)=1.55$, n.s. $]$, no main effect of speed $[F(1,3)=2.88$, n.s. $]$, and no interaction. To ensure that there was no ability to see monocular motion in the

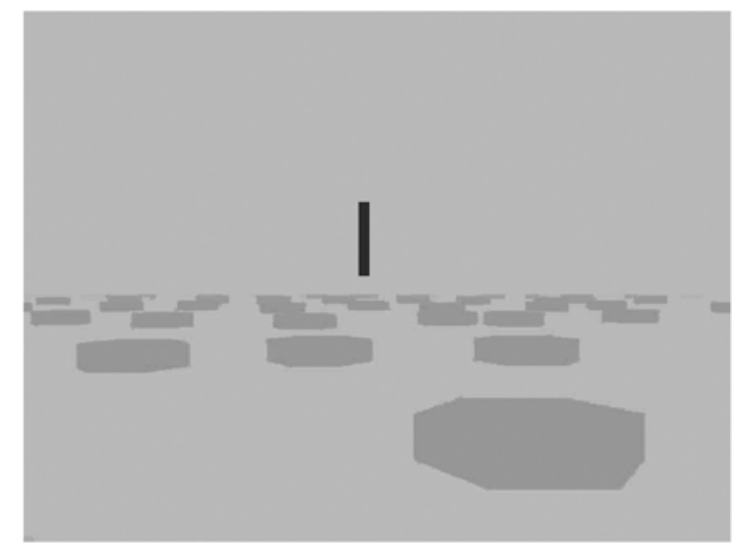

Figure 3. Optic flow stimulus used in the equated motion visibility heading experiment (a reduced contrast version of the original optic flow stimulus). The final frame is depicted. The target line was not present during the simulated self-motion. 

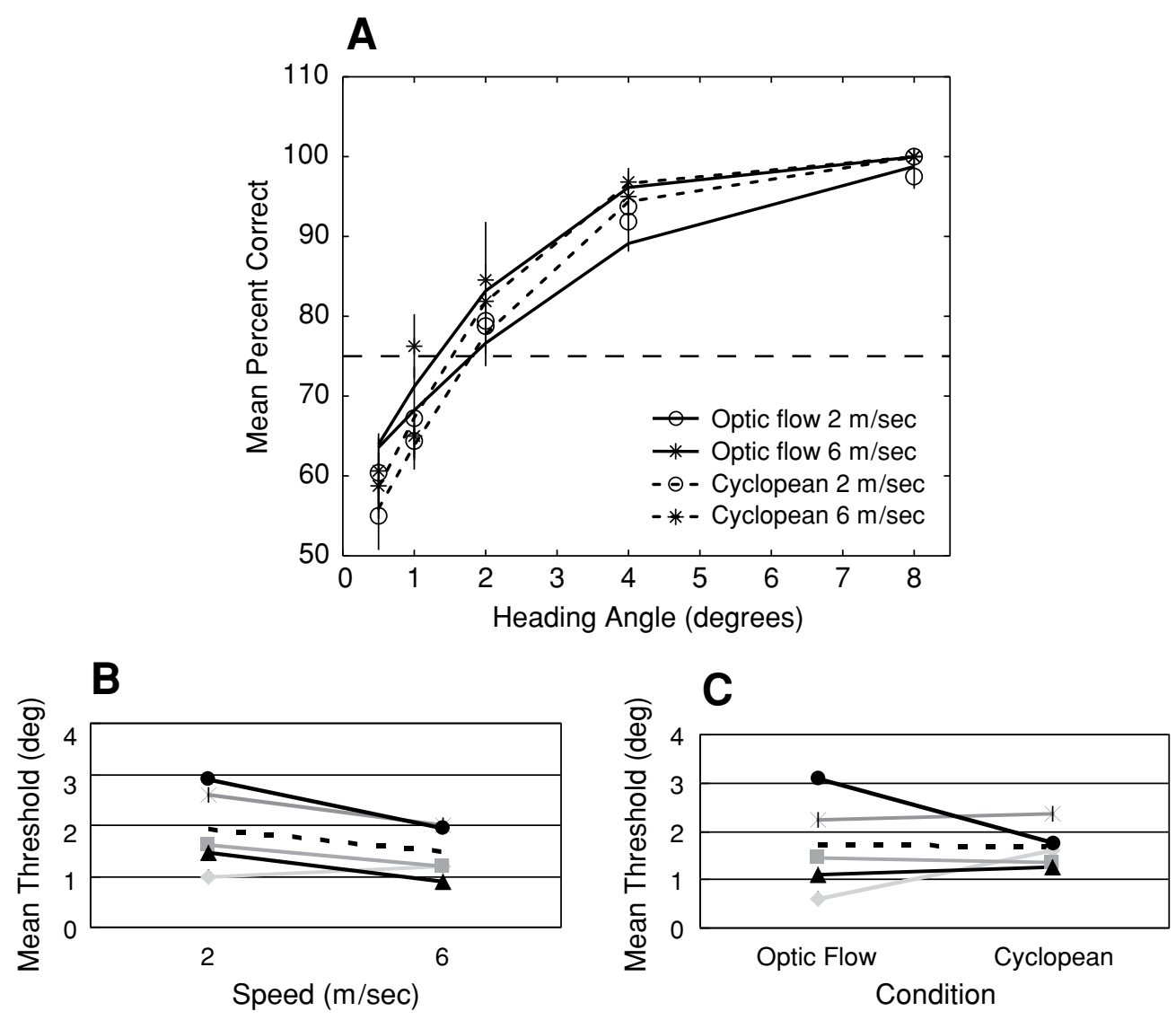

Figure 4. Results for the equated motion visibility heading experiment. (A) Mean percent correct for 5 observers for each speed in each display condition. Speed was crossed with display condition, and data were collapsed across positive and negative heading angles. (B) Individual subjects' mean threshold values for each display condition, collapsed across speed. The dashed line depicts average threshold values. (C) Individual subjects' mean threshold values for each speed, collapsed across display condition. The dashed line depicts average threshold values.

cyclopean stimulus, we included a control experiment to check for monocular artifacts.

\section{Control Experiment to Rule Out the Presence of Monocular Cues in Cyclopean Stimulus}

An additional control experiment was conducted with 4 naive subjects to allay concerns that any monocular artifacts in the scintillating pattern of dots presented to each eye were responsible for the impressive cyclopean thresholds. In a monocular cyclopean control condition, subjects viewed the cyclopean stimulus with one eye covered while making heading judgments. Subjects also performed the heading task while viewing the cyclopean stimulus binocularly, just as subjects did in the main experiment. Procedures were also identical. Mean thresholds of $75 \%$ correct were $1.8^{\circ}$ for the binocular cyclopean stimulus. However, monocular cyclopean thresholds could not be obtained. The monocular cyclopean heading data were best fit by a straight line that intercepts the $y$-axis (mean percent correct) at $45 \%$ and has a slope of less than 0.1 . Thus in this control condition, subjects performed no bet- ter than chance, indicating that no exploitable monocular artifact was present in the cyclopean stimulus. Therefore, any concern that heading judgments in the cyclopean condition could have been supported by a monocular artifact can be eliminated. In fact, every one of the subjects, when asked afterward about their performance, noted that the monocular cyclopean control condition seemed a futile and tedious exercise.

\section{GENERAL DISCUSSION}

Given the comparable performance with both cyclopean and optic flow stimuli, we conclude that heading perception is not fundamentally dependent on retinal optic flow. In a relevant debate concerning active steering, Rushton, Harris, Lloyd, and Wann (1998) championed the visual direction strategy and demonstrated, by pitting it against a perceived heading strategy using optic flow, that visual direction is predominantly used for steering (see also Wann \& Land, 2000). Research since then has established a role for both visual direction and perceived heading from 
optic flow, depending on the availability and richness of cues (Warren, Kay, Zosh, Duchon, \& Sahuc, 2001; Wood, Harvey, Young, Beedie, \& Wilson, 2000). Here, we are investigating a different question: Is optic flow even necessary for perceiving heading? We have shown here that the answer is no.

Nevertheless, the assumption that optic flow is used has been incorporated into many computational models (Heeger \& Jepson, 1992; Hildreth, 1992; Koenderink \& van Doorn, 1987; Longuet-Higgins \& Prazdny, 1980; Rieger \& Lawton, 1985; and for a review, see Lappe, 2000). Our results imply that models that are formulated exclusively on retinal optical flow may be somewhat limited in scope. Here we have demonstrated that cyclopean stimuli allow for accurate heading judgments. Subjects are able to use changes in the disparity structure of the scene to determine their heading. As such, models would benefit from including stereoscopic input in addition to retinal optical flow, or from considering a more abstract type of flow that is computed at or beyond the site of binocular combination. Lappe (1996) presented a model that integrates static stereoscopic depth cues, although this model uses such cues only to reduce the noise in optic flow.

Early neurophysiological studies of cortical area MST revealed areas selective to optic flow stimuli (Duffy \& Wurtz, 1991; Saito et al., 1986; Tanaka et al., 1986), providing some support for the conventional view that optic flow is a fundamental source of information for heading perception. However, area MST receives information from the middle temporal (MT) area, which has cells sensitive to both motion and disparity (Bradley, Qian, \& Andersen, 1995; DeAngelis \& Newsome, 1999; Maunsell \& Van Essen, 1983). Additionally, disparity-dependent neurons in area MST are capable of specifying the direction of selfmotion (Roy et al., 1992; Roy \& Wurtz, 1990). Thus, areas MT and MST have both motion- and disparity-selective neurons. The role of stereoscopic cues in heading perception has previously been discussed in terms of static disparity cues disambiguating optic flow (discounting the rotational components of flow due to eye rotations) to improve performance (van den Berg \& Brenner, 1994). Perhaps the role of optic flow has been exaggerated and the importance of binocular information neglected. This question could be examined with experiments using the cyclopean stimuli presented here. Source code for displaying cyclopean stimuli is available at www.recveb.ucsb.edu/sticky dots.htm. We do not yet know how cyclopean information is processed or combined with optic flow information in order to determine heading. Perhaps there is one process that is sensitive to both stereoscopic and optic flow based motion in depth, a more abstract flow. Another possibility is that disparity information and optic flow information are computed and used independently.

\section{Conclusion}

Since Gibson's insight outlining the use of optic flow to control heading, research on this topic has flourished. Though psychophysical research has demonstrated the sufficiency of retinal optic flow for heading detection, it has failed to establish its necessity for such judgments. Here, we eschew the idea that a prototypical optic flow field is essential for accurate heading estimation. Heading is not directly and solely computed by a mechanism that processes only retinal optic flow, since heading can be determined from other visual information as well. In these experiments, we demonstrate that heading can be computed from changing binocular disparities, which provide no retinal optic flow to the observer.

\section{REFERENCES}

Beusmans, J. M. H. (1998). Perceived object shape affects the perceived direction of self-movement. Perception, 27, 1079-1085.

Bradley, D. C., Qian, N., \& Andersen, R. A. (1995). Integration of motion and stereopsis in middle temporal cortical area of macaques. Nature, 373, 609-611.

Bremmer, F., Duhamel, J.-R., Hamed, S. B., \& Graf, W. (2000). Stages of self-motion processing in primate posterior parietal cortex. International Review of Neurobiology, 44, 173-198.

Britten, K. H., \& VAN WeZel, R. J. A. (1998). Electrical microstimulation of cortical area MST biases heading perception in monkeys. Nature Neuroscience, 1, 59-63.

Cutting, J. E., Springer, K., Braren, P. A., \& Johnson, S. H. (1992). Wayfinding on foot from information in retinal, not optical, flow. Journal of Experimental Psychology: General, 121, 41-72.

DeAngelis, G. C., \& Newsome, W. T. (1999). Organization of disparityselective neurons in macaque area MT. Journal of Neuroscience, 19, 1398-1415.

Donnelly, M., Bowd, C., \& Patterson, R. (1997). Direction discrimination of cyclopean (stereoscopic) and luminance motion. Vision Research, 37, 2041-2046.

Duffy, C. J., \& WurTz, R. H. (1991). Sensitivity of MST neurons to optic flow stimuli: I. A continuum of response selectivity to large-field stimuli. Journal of Neurophysiology, 65, 1329-1345.

GiBson, J. J. (1950). Perception of the visual world. Boston: Houghton Mifflin.

GiBson, J. J. (1958). Visually controlled locomotion and visual orientation in animals. British Journal of Psychology, 49, 182-194.

GraY, R., \& REgAN, D. (1996). Cyclopean motion perception produced by oscillations of size, disparity and location. Vision Research, 36, 655-665.

Hahn, S., Andersen, G. J., \& SaIdpour, A. (2003). Static scene analysis for the perception of heading. Psychological Science, 14, 543-548.

HeEger, D. J., \& Jepson, A. D. (1992). Subspace methods for recovering rigid motion I: Algorithm and implementation. International Journal of Computer Vision, 7, 95-117.

HiLDRETH, E. C. (1992). Recovering heading for visually-guided navigation. Vision Research, 32, 1177-1192.

JuLESZ, B. (1971). Foundations of cyclopean perception. Chicago: University of Chicago Press.

Koenderink, J. J., \& van Doorn, A. J. (1987). Facts on optic flow. Biological Cybernetics, 56, 247-254.

LAPPE, M. (1996). Functional consequences of an integration of motion and stereopsis in area MT of monkey extrastriate visual cortex. Neural Computation, 8, 1449-1461.

LAPPE, M. (2000). Computational mechanisms for optic flow analysis in primate cortex. International Review of Neurobiology, 44, 235-268.

Longuet-Higgins, H. C., \& Prazdny, K. (1980). The interpretation of a moving retinal image. Proceedings of the Royal Society of London: Series B, 208, 385-397.

Maunsell, J. H., \& VAn Essen, D. C. (1983). Functional properties of neurons in middle temporal visual area of the macaque monkey: II. Binocular interactions and sensitivity to binocular disparity. Journal of Neurophysiology, 49, 1148-1167.

Palmisano, S. (1996). Perceiving self-motion in depth: The role of stereoscopic motion and changing-size cues. Perception \& Psychophysics, 58, 1168-1176. 
Patterson, R. (1999). Stereoscopic (cyclopean) motion sensing. Vision Research, 39, 3329-3345.

Portfors-Yeomans, C. V., \& Regan, D. (1996). Cyclopean discrimination thresholds for the direction and speed of motion in depth. Vision Research, 36, 3265-3279.

RAFFI, M., \& SiEgel, R. M. (2004). Multiple cortical representations of optic flow processing. In L. Vaina, S. Beardsley, \& S. Rushton (Eds.), Optic flow and beyond (pp. 3-22). Boston: Kluwer.

Rieger, J. H., \& Lawton, D. T. (1985). Processing differential image motion. Journal of the Optical Society of America A, 2, 354-360.

Roy, J. P., Komatsu, H., \& Wurtz, R. H. (1992). Disparity sensitivity of neurons in monkey extrastriate area MST. Journal of Neuroscience, 12, 2478-2492.

Roy, J. P., \& Wurtz, R. H. (1990). The role of disparity-sensitive cortical neurons in signalling the direction of self-motion. Nature, $\mathbf{3 4 8}$ 160-162.

Royden, C. S., Banks, M. S., \& Crowell, J. A. (1992). The perception of heading during eye movements. Nature, 360, 583-585.

Rushton, S. K., Harris, J. M., Lloyd, M. R., \& WanN, J. P. (1998). Guidance of locomotion on foot uses perceived target location rather than optic flow. Current Biology, 8, 1191-1194.

Saito, H., Yukie, M., Tanaka, K., Hikosaka, K., Fukada, Y., \& Iwai, E. (1986). Integration of direction signals of image motion in the superior temporal sulcus of the macaque monkey. Journal of Neuroscience, $\mathbf{6}$, 145-157.

Schrater, P. R., Knill, D. C., \& Simoncelli, E. P. (2001). Perceiving visual expansion without optic flow. Nature, 410, 816-819.
TanaKa, K., Hikosaka, K., Saito, H., Yukie, M., Fukada, Y., \& Iwai, E. (1986). Analysis of local and wide-field movements in the superior temporal visual areas of the macaque monkey. Journal of Neuroscience, 6, 134-144.

van den Berg, A. V. (1993). Perception of heading. Nature, 365, 497498

VAN DEN Berg, A. V., \& Brenner, E. (1994). Why two eyes are better than one for judgments of heading. Nature, 371, 700-702.

WanN, J. P., \& LAND, M. F. (2000). Steering with or without the flow: Is the retrieval of heading necessary? Trends in Cognitive Sciences, 4, 319-324.

WARren, W. H., JR., \& HANnON, D. J. (1988). Direction of self-motion is perceived from optical flow. Nature, 336, 162-163.

Warren, W. H., Jr., KaY, B. A., Zosh, W. D., Duchon, A. P., \& SAHuc, S. (2001). Optic flow is used to control human walking. Nature Neuroscience, 4, 213-216.

Warren, W. H., Jr., Morris, M. W., \& Kalish, M. (1988). Perception of translational heading from optical flow. Journal of Experimental Psychology: Human Perception \& Performance, 14, 646-660.

Wood, R. M., Harvey, M. A., Young, C. E., Beedie, A., \& Wilson, T. (2000). Weighting to go with the flow? Current Biology, 10, R545R546.

(Manuscript received August 26, 2004; revision accepted for publication August 24, 2005.) 